OPEN ACCESS

Edited by:

Bin Jiang,

Peking University, China

Reviewed by:

Robert Drury

Canary Systems, United States

Nan Huo,

Mayo Clinic, United States

*Correspondence:

Minghui Li

mli54@uthsc.edu

${ }^{\dagger}$ These authors have contributed equally to this work

Specialty section:

This article was submitted to Family Medicine and Primary Care, a section of the journal

Frontiers in Medicine

Received: 27 February 2021 Accepted: 17 May 2021

Published: 09 June 2021

Citation:

Lv G, Yuan J, Hsieh S, Shao R and Li M (2021) Knowledge and Determinants of Behavioral Responses to the Pandemic of COVID-19. Front. Med. 8:673187. doi: 10.3389/fmed.2021.673187

\section{Knowledge and Determinants of Behavioral Responses to the Pandemic of COVID-19}

\author{
Gang $\mathrm{Lv}^{1 \dagger}$, Jing Yuan ${ }^{2 \dagger}$, Stephanie Hsieh ${ }^{3}$, Rongjie Shao and Minghui $\mathrm{Li}^{5 *}$ \\ ${ }^{1}$ Department of General Surgery, The 1st Medical Center of Chinese PLA General Hospital, Beijing, China, ${ }^{2}$ Department of \\ Clinical Pharmacy and Pharmacy Administration, School of Pharmacy, Fudan University, Shanghai, China, ${ }^{3}$ Department of \\ Pharmacy, Scarborough Health Network - Centenary Hospital, Scarborough, ON, Canada, ${ }^{4}$ Department of Health \\ Economics, China Pharmaceutical University, Nanjing, China, ${ }^{5}$ Department of Clinical Pharmacy and Translational Science, \\ University of Tennessee Health Science Center, Memphis, TN, United States
}

Background: Understanding knowledge and behavioral responses to the pandemic of coronavirus disease 2019 (COVID-19) is important for appropriate public health interventions.

Objectives: To assess knowledge of COVID-19 and to examine determinants associated with the adoption of preventive health behaviors among future health care providers.

Methods: An anonymous online survey was sent out to pharmacy students in high and low-endemic areas of COVID-19 in China. Based on recommendations from the Chinese Center for Disease Control and Prevention, preventive health behaviors examined in this study included washing hands, wearing a face mask, and maintaining social distancing. The Health Belief Model (HBM) was used and measured by a seven-point Likert scale (one as extremely unlikely; seven as extremely likely). Multivariate linear regression models were used to examine predictors of preventive health behaviors.

Results: Among 203 respondents who finished the survey, a medium level of knowledge (4.41 \pm 0.95$)$ of COVID-19 was reported. Respondents were extremely likely to wear a face mask (6.85 \pm 0.60 ), but only moderately likely to engage in washing hands $(5.95 \pm 1.38)$ and maintaining social distancing (6.19 \pm 1.60$)$. Determinants of washing hands were cue to action, self-efficacy, knowledge, and gender; wearing a face mask were cue to action, self-efficacy, knowledge, and ethnicity; and maintaining social distancing were cue to action and self-efficacy.

Conclusions: Public health interventions should consider incorporating cue to action, self-efficacy, and knowledge as factors to potentially improve the adoption of face mask-wearing, hand washing, and social distancing as appropriate individual preventive measures, especially if local and regional authorities are considering reopening schools sometime in future.

Keywords: COVID-19, knowledge, determinants of health, preventive health behaviors, pharmacist, student 


\section{INTRODUCTION}

The coronavirus disease 2019 (COVID-19) is a new, rapidly spreading infectious disease caused by the "SARS-CoV-2" virus. World Health Organization (WHO) has declared COVID-19 as a pandemic (1). The first case of COVID-19 was reported in Wuhan, China in December 2019 (1). Since then, it has spread globally at an alarming rate. National and regional authorities have implemented different prevention and control measures to contain the quick spread of COVID-19. Individuals also have different behavioral responses to the pandemic of COVID-19.

Government responses to COVID-19 have included implementing lockdowns, canceling public gatherings, imposing travel restrictions, and closing schools. The effectiveness of these measures has been debated in the literature. The implementation of lockdown and travel restrictions was found to be effective in reducing new infections $(2,3)$. School closures might lead to a reduction in mortality (4). Restricting travels from areas with active outbreaks could reduce disease transmission if combined with public health interventions and individual behavior changes (5).

To encourage individual behavior changes during the pandemic of COVID-19, both local and global health organizations have released recommendations on individual preventive measures to reduce disease transmissibility. World Health Organization recommends frequent hand washing, social distancing, avoidance of face touching, practicing respiratory hygiene, staying home, and seeking medical care early if there are suspected symptoms (6). The Centers for Disease Control and Prevention (CDC) in different countries have similar recommendations on hand washing and social distancing, but have different opinions on face mask-wearing $(7,8)$. However, little is known about whether individuals have changed their behaviors based on recommendations from health organizations.

This study used the Health Belief Model (HBM) as the conceptual framework. The HBM is one of the most widely used psychosocial models to examine factors associated with individual behavior (9). Key components of the HBM included perceived susceptibility (the risk of getting a disease), perceived severity (the seriousness of a disease), perceived benefits (the gains to perform a behavior), perceived barriers (the obstacles to perform a behavior), and cue to action (the stimulus to perform a behavior) (9). Recent modifications of the HBM have added other components of self-efficacy (the level of confidence to perform a behavior) and knowledge (the level of expertise in an area) $(10,11)$.

The HBM has been used to assess predictors of adopting preventive health behaviors in previous infectious disease outbreaks. Perceived susceptibility significantly influenced the adoption of preventive health behaviors during the severe acute respiratory syndrome (SARS) and H1N1 outbreaks (12-15). Perceived severity significantly influenced the adoption of preventive health behaviors during the H1N1 outbreak (16, 17). Perceived benefits significantly influenced the adoption of preventive health behaviors during the SARS and H1N1 outbreaks $(12,14-17)$. Perceived barriers significantly influenced the adoption of preventive health behaviors during the H1N1 outbreak (15-17). Cues to action significantly influenced the adoption of preventive health behaviors during the SARS, H1N1, and Zika virus outbreaks $(12-15,18)$. Self-efficacy was not associated with the adoption of preventive health behaviors during the SARS and H1N1 outbreaks $(13,15)$. Knowledge did not influence the adoption of preventive health behaviors during the H1N1 and SARS outbreak $(13,16)$.

Understanding individuals' knowledge and behavioral responses to the pandemic of COVID-19 is important to contain the outbreak. However, there is a paucity of studies available on the knowledge and preventive health behaviors of COVID-19. Therefore, to address these literature gaps, this study aimed to (1) to assess individuals' knowledge of COVID-19 and (2) to examine determinants associated with the potential adoption of preventive health behaviors, including washing hands, wearing a face mask, and maintaining social distancing, among future pharmacists.

\section{METHODS}

\section{Study Participants}

This study was conducted in March 2020 in China. During that time, China had slowed the spread of COVID-19 and saw continuous decreases in the newly confirmed cases. In low-endemic areas, most prevention and control measures implemented by local authorities were discontinued (e.g., lockdowns and travel restrictions). However, school closures were still in effect in most parts of China. Specifically, all colleges and universities were still closed and classes were offered online. A significant challenge for schools to reopen is that students might not have enough knowledge and motivation to engage in preventive health behaviors to prevent contracting and spreading COVID-19 in the community.

A pilot study was conducted on eight respondents to test the clarity of survey questions and confusing questions were revised. An anonymous online survey was sent to pharmacy students who lived in high and low-endemic areas in China. We sent surveys to 800 pharmacy students who were 18 years and older from four different colleges/universities, including both comprehensive and professional colleges/universities. High-endemic areas included Hubei, Guangdong, Henan, Zhejiang, and Hunan, where the total number of COVID-19 cases were 1,000 and higher; low-endemic areas included provinces with the total number of COVID-19 cases lower than 1,000. This study was approved by the University institutional review board.

\section{Measurement}

The survey collected information on knowledge of COVID-19, preventive health behaviors, and demographics. Respondents' knowledge was assessed by when and how they first heard of COVID-19. This study also tested if respondents knew how COVID-19 was transmitted and of the availability of test kit, medication, and vaccine for COVID-19. Demographics collected in the survey included age, gender, ethnicity, and residence area (high vs. low-endemic areas).

Preventive health behaviors examined in this study included washing hands, wearing a face mask, and maintaining social 
distancing as recommended by the China CDC (8). Respondents were asked to indicate their likelihood of performing these preventive health behaviors and measured by a seven-point Likert scale (one as extremely unlikely; seven as extremely likely). Components of the HBM were also measured by a seven-point Likert scale (one as strongly disagree; seven as strongly agree). Perceived susceptibility was assessed by whether respondents believe that they are at risk of getting COVID-19. Perceived severity was assessed by their thoughts of COVID-19 as a serious illness. Perceived benefits were assessed by whether respondents believe each preventive health behavior can prevent COVID-19. Perceived barriers were assessed by whether they believe it is inconvenient to perform each preventive health behavior. Cue to action was assessed by whether respondents would perform each preventive health behavior if recommended. Self-efficacy was assessed by whether they are able to perform each preventive health behavior. Knowledge was assessed by whether respondents have enough knowledge about COVID-19 and defined as low (score 1-2), medium (score 3-5), and high (score 6-7) levels.

\section{Data Analysis}

We excluded study participants in the data analysis if they reported extreme answers, defined as having the same response to all survey questions. Preventive health behaviors and components of the HBM were analyzed as means and compared between different demographic groups using independent sample $t$-tests. Pearson correlation coefficients were calculated to examine associations between different preventive health behaviors. To predict preventive health behaviors, three multivariate linear regression models were used. In each model, the dependent variable was the preventive health behavior and independent variables included components of the HBM and demographic variables. All statistical analyses were conducted using SAS version 9.4 (SAS Institute, Cary, NC).

\section{RESULTS}

Among 203 respondents who finished the survey (response rate of $25.38 \%$ ), the majority of them were aged $18-22$ years (54.68\%), female (69.95\%), of Han ethnicity (91.13\%), and lived in low-endemic areas (70.44\%) (Table 1). For the knowledge of COVID-19, most of respondents first heard of COVID-19 in January 2020 (59.61\%). The two most common sources of this information were websites $(84.73 \%)$ and social media (59.11\%). Almost all respondents knew that COVID-19 was transmitted through droplets $(98.52 \%)$ and two-thirds of them also knew that close contact $(66.50 \%)$ could transmit the virus. For the availability of different medical products, the majority of respondents thought that we have test kits to detect $(79.31 \%)$ but do not have a vaccine to prevent COVID-19 (78.82\%). Approximately half of respondents thought that there is no medication to treat COVID-19 (52.22\%).

For preventive health behaviors, respondents reported being extremely likely to wear a face mask $(6.85 \pm 0.60)$, but only moderately likely to engage in hand washing $(5.95 \pm 1.38)$ and social distancing (6.19 \pm 1.60$)$ (Table 2). For Pearson correlation coefficients, three preventive health behaviors were positively
TABLE 1 | Characteristics and knowledge of study respondents $(N=203)$.

\begin{tabular}{|c|c|c|}
\hline & $N$ & $\%$ \\
\hline \multicolumn{3}{|l|}{ Demographics } \\
\hline \multicolumn{3}{|l|}{ Age } \\
\hline 18-22 & 111 & 54.68 \\
\hline $23+$ & 92 & 45.32 \\
\hline \multicolumn{3}{|l|}{ Gender } \\
\hline Female & 142 & 69.95 \\
\hline Male & 61 & 30.05 \\
\hline \multicolumn{3}{|l|}{ Ethnicity } \\
\hline Han & 185 & 91.13 \\
\hline Minority & 18 & 8.87 \\
\hline \multicolumn{3}{|l|}{ Area } \\
\hline Low-endemic area & 143 & 70.44 \\
\hline High-endemic area & 60 & 29.56 \\
\hline \multicolumn{3}{|c|}{ First Time Heard of COVID-19 } \\
\hline 2019 & 75 & 36.95 \\
\hline January 2020 & 121 & 59.61 \\
\hline February 2020 and later & 7 & 3.45 \\
\hline \multicolumn{3}{|l|}{ Source of Information } \\
\hline $\mathrm{TV}$ & 49 & 24.14 \\
\hline Website & 172 & 84.73 \\
\hline Social media & 120 & 59.11 \\
\hline Radio & 9 & 4.43 \\
\hline In print & 7 & 3.45 \\
\hline Family/friend & 54 & 26.60 \\
\hline \multicolumn{3}{|l|}{ Method of Transmission } \\
\hline Close contact & 135 & 66.50 \\
\hline Droplets & 200 & 98.52 \\
\hline Bodily fluids & 90 & 44.33 \\
\hline Sex & 79 & 38.92 \\
\hline \multicolumn{3}{|l|}{ Availability of Test Kit } \\
\hline Yes & 161 & 79.31 \\
\hline No & 23 & 11.33 \\
\hline Unsure & 19 & 9.36 \\
\hline \multicolumn{3}{|l|}{ Availability of Medication } \\
\hline Yes & 56 & 27.59 \\
\hline No & 106 & 52.22 \\
\hline Unsure & 41 & 20.20 \\
\hline \multicolumn{3}{|l|}{ Availability of Vaccine } \\
\hline Yes & 15 & 7.39 \\
\hline No & 160 & 78.82 \\
\hline Unsure & 28 & 13.79 \\
\hline
\end{tabular}

correlated with each other (washing hands vs. wearing a face mask: $r=0.24, p<0.01$; washing hands vs. maintaining social distancing: $r=0.24, p<0.01$; wearing a face mask vs. maintaining social distancing: $r=0.31, p<0.01$ ). Different demographic groups had a similar likelihood of performing preventive health behaviors.

For components of the HBM, respondents reported a slightly low perceived susceptibility $(2.92 \pm 1.70)$ of COVID-19 and males reported a lower level of susceptibility (Table 2). 
TABLE 2 | Preventive health behaviors of study respondents by age, gender, ethnicity, and residence area.

\begin{tabular}{|c|c|c|c|c|c|c|c|c|c|c|c|c|c|}
\hline & \multirow{2}{*}{$\begin{array}{c}\text { Overall } \\
\text { Mean } \pm \mathbf{S D}^{\dagger}\end{array}$} & \multicolumn{3}{|c|}{ Age } & \multicolumn{3}{|c|}{ Gender } & \multicolumn{3}{|c|}{ Ethnicity } & \multicolumn{3}{|c|}{ Residence area } \\
\hline & & $\begin{array}{c}18-22 \\
\text { Mean } \pm \text { SD }\end{array}$ & $\begin{array}{c}23+ \\
\text { Mean } \pm \text { SD }\end{array}$ & $P$ & $\begin{array}{c}\text { Female } \\
\text { Mean } \pm \text { SD }\end{array}$ & $\begin{array}{c}\text { Male } \\
\text { Mean } \pm \text { SD }\end{array}$ & $P$ & $\begin{array}{c}\text { Han } \\
\text { Mean } \pm \text { SD }\end{array}$ & $\begin{array}{c}\text { Minority } \\
\text { Mean } \pm \text { SD }\end{array}$ & $\boldsymbol{P}$ & $\begin{array}{c}\text { Low-endemic } \\
\text { Mean } \pm \text { SD }\end{array}$ & $\begin{array}{c}\text { High-endemic } \\
\text { Mean } \pm \text { SD }\end{array}$ & $P$ \\
\hline \multicolumn{14}{|l|}{ Preventive health behavior of } \\
\hline Washing hands & $5.95 \pm 1.38$ & $6.02 \pm 1.29$ & $5.87 \pm 1.48$ & & $6.02 \pm 1.34$ & $5.79 \pm 1.45$ & & $5.91 \pm 1.40$ & $6.33 \pm 1.03$ & & $5.94 \pm 1.37$ & $5.97 \pm 1.41$ & \\
\hline Wearing a face mask & $6.85 \pm 0.60$ & $6.88 \pm 0.51$ & $6.82 \pm 0.69$ & & $6.82 \pm 0.64$ & $6.90 \pm 0.47$ & & $6.86 \pm 0.57$ & $6.72 \pm 0.83$ & & $6.85 \pm 0.54$ & $6.83 \pm 0.71$ & \\
\hline Maintaining social distancing & $6.19 \pm 1.60$ & $6.39 \pm 1.36$ & $5.96 \pm 1.84$ & * & $6.23 \pm 1.57$ & $6.11 \pm 1.68$ & & $6.23 \pm 1.56$ & $5.83 \pm 2.04$ & & $6.14 \pm 1.63$ & $6.32 \pm 1.56$ & \\
\hline Perceived susceptibility of COVID-19 & $2.92 \pm 1.70$ & $3.05 \pm 1.72$ & $2.75 \pm 1.67$ & & $3.08 \pm 1.65$ & $2.52 \pm 1.77$ & $\star *$ & $2.91 \pm 1.70$ & $3.00 \pm 1.78$ & & $2.88 \pm 1.68$ & $3.00 \pm 1.76$ & \\
\hline Perceived severity of COVID-19 & $5.08 \pm 1.51$ & $5.00 \pm 1.50$ & $5.17 \pm 1.53$ & & $4.96 \pm 1.46$ & $5.36 \pm 1.60$ & * & $5.11 \pm 1.53$ & $4.72 \pm 1.27$ & & $5.17 \pm 1.46$ & $4.85 \pm 1.61$ & \\
\hline \multicolumn{14}{|l|}{ Perceived benefits of } \\
\hline Washing hands & $5.50 \pm 1.27$ & $5.47 \pm 1.23$ & $5.54 \pm 1.32$ & & $5.44 \pm 1.29$ & $5.64 \pm 1.24$ & & $5.50 \pm 1.22$ & $5.50 \pm 1.76$ & & $5.51 \pm 1.19$ & $5.48 \pm 1.47$ & \\
\hline Wearing a face mask & $5.87 \pm 1.23$ & $5.97 \pm 1.15$ & $5.74 \pm 1.32$ & & $5.83 \pm 1.24$ & $5.95 \pm 1.22$ & & $5.82 \pm 1.26$ & $6.33 \pm 0.77$ & $\star \star$ & $5.86 \pm 1.19$ & $5.88 \pm 1.33$ & \\
\hline Maintaining social distancing & $6.19 \pm 0.93$ & $6.25 \pm 0.86$ & $6.11 \pm 1.01$ & & $6.18 \pm 0.95$ & $6.20 \pm 0.89$ & & $6.18 \pm 0.91$ & $6.22 \pm 1.12$ & & $6.18 \pm 0.95$ & $6.20 \pm 0.88$ & \\
\hline \multicolumn{14}{|l|}{ Perceived barriers to } \\
\hline Washing hands & $2.65 \pm 1.69$ & $2.47 \pm 1.63$ & $2.87 \pm 1.76$ & * & $2.68 \pm 1.63$ & $2.59 \pm 1.86$ & & $2.73 \pm 1.72$ & $1.78 \pm 1.06$ & 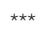 & $2.62 \pm 1.71$ & $2.72 \pm 1.67$ & \\
\hline Wearing a face mask & $5.17 \pm 1.66$ & $5.17 \pm 1.68$ & $5.17 \pm 1.64$ & & $5.23 \pm 1.57$ & $5.03 \pm 1.86$ & & $5.26 \pm 1.60$ & $4.22 \pm 2.02$ & $\star \star$ & $5.13 \pm 1.70$ & $5.28 \pm 1.56$ & \\
\hline Maintaining social distancing & $2.58 \pm 1.76$ & $2.33 \pm 1.68$ & $2.87 \pm 1.83$ & $\star *$ & $2.63 \pm 1.75$ & $2.46 \pm 1.79$ & & $2.59 \pm 1.75$ & $2.39 \pm 1.88$ & & $2.57 \pm 1.76$ & $2.60 \pm 1.78$ & \\
\hline \multicolumn{14}{|l|}{ Cue to action of } \\
\hline Washing hands & $6.27 \pm 1.03$ & $6.38 \pm 1.01$ & $6.13 \pm 1.04$ & * & $6.27 \pm 1.03$ & $6.26 \pm 1.03$ & & $6.23 \pm 1.06$ & $6.61 \pm 0.61$ & $\star \star$ & $6.24 \pm 0.99$ & $6.32 \pm 1.13$ & \\
\hline Wearing a face mask & $6.59 \pm 0.72$ & $6.69 \pm 0.55$ & $6.47 \pm 0.87$ & ** & $6.57 \pm 0.69$ & $6.64 \pm 0.80$ & & $6.57 \pm 0.73$ & $6.78 \pm 0.55$ & & $6.56 \pm 0.75$ & $6.67 \pm 0.65$ & \\
\hline Maintaining social distancing & $6.28 \pm 1.18$ & $6.29 \pm 1.27$ & $6.26 \pm 1.07$ & & $6.17 \pm 1.29$ & $6.52 \pm 0.83$ & ** & $6.28 \pm 1.15$ & $6.22 \pm 1.52$ & & $6.28 \pm 1.13$ & $6.27 \pm 1.29$ & \\
\hline \multicolumn{14}{|l|}{ Self-efficacy of } \\
\hline Washing hands & $5.84 \pm 1.30$ & $5.95 \pm 1.26$ & $5.71 \pm 1.34$ & & $5.84 \pm 1.33$ & $5.84 \pm 1.24$ & & $5.79 \pm 1.32$ & $6.33 \pm 0.91$ & * & $5.80 \pm 1.23$ & $5.93 \pm 1.50$ & \\
\hline Wearing a face mask & $6.60 \pm 0.70$ & $6.70 \pm 0.53$ & $6.47 \pm 0.84$ & ** & $6.59 \pm 0.73$ & $6.61 \pm 0.64$ & & $6.58 \pm 0.72$ & $6.78 \pm 0.43$ & * & $6.61 \pm 0.65$ & $6.57 \pm 0.81$ & \\
\hline Maintaining social distancing & $6.07 \pm 1.13$ & $6.17 \pm 1.04$ & $5.95 \pm 1.23$ & & $6.08 \pm 1.16$ & $6.03 \pm 1.06$ & & $6.05 \pm 1.11$ & $6.28 \pm 1.32$ & & $6.06 \pm 1.15$ & $6.08 \pm 1.09$ & \\
\hline Knowledge of COVID-19 & $4.41 \pm 0.95$ & $4.32 \pm 0.94$ & $4.52 \pm 0.95$ & & $4.30 \pm 0.86$ & $4.69 \pm 1.09$ & $\star *$ & $4.38 \pm 0.94$ & $4.72 \pm 0.96$ & & $4.44 \pm 0.98$ & $4.35 \pm 0.88$ & \\
\hline
\end{tabular}

${ }^{\dagger} 1$ as extremely unlikely and 7 as extremely likely. ${ }^{*}$ Significant at $0.1,{ }^{* *}$ Significant at $0.05,{ }^{* * *}$ Significant at 0.01 . 
Respondents reported a slightly high perceived severity (5.08 \pm 1.51 ) of COVID-19. Perceived benefits were moderately high for all preventive health behaviors (washing hands: 5.50 \pm 1.27 ; wearing a face mask: $5.87 \pm 1.23$; and maintaining social distancing: $6.19 \pm 0.93$ ) and ethnic minorities reported a higher level of benefit of wearing a face mask. Perceived barriers were slightly low to wash hands $(2.65 \pm 1.69)$ and maintain social distancing $(2.58 \pm 1.76)$. Ethnic minorities reported a lower level of barrier to wash hands and those aged 23 years and older reported a higher level of barrier to maintain social distancing. Perceived barriers to wear a face mask were slightly high $(5.17 \pm 1.66)$ and ethnic minorities reported a lower level of barrier. Cue to action was moderately high for washing hands $(6.27 \pm 1.03)$ and maintaining social distancing $(6.28 \pm 1.18)$. Ethnic minorities reported a higher level of cue to action of washing hands and males reported a higher level of cue to action of maintaining social distancing. Cue to action of wearing a face mask was extremely high $(6.59 \pm 0.72)$ and those aged 23 years and older reported a lower level of cue to action. Self-efficacy was moderately high for washing hands $(5.84 \pm 1.30)$ and maintaining social distancing $(6.07 \pm 1.13)$. Self-efficacy of wearing a face mask was extremely high $(6.60 \pm 0.70)$ and those aged 23 years and older reported a lower level of self-efficacy. Respondents reported a medium level of knowledge (4.41 \pm 0.95$)$ of COVID-19 and males reported a higher level of knowledge.

In the multivariate regression models, determinants of washing hands included cue to action ( $\beta$ : $0.27 ; 95 \% \mathrm{CI}: 0.05$ to 0.48 ), self-efficacy ( $\beta: 0.56 ; 95 \%$ CI: 0.39 to 0.73 ), and knowledge $(\beta: 0.15 ; 95 \%$ CI: 0.01 to 0.31$)$ and being a male was negatively associated with washing hands $(\beta$ : $-0.35 ; 95 \% \mathrm{CI}:-0.68$ to -0.03 ) (Table 3). Determinants of wearing a face mask included cue to action ( $\beta: 0.20 ; 95 \%$ CI: 0.06 to 0.34$)$, self-efficacy $(\beta: 0.21$; 95\% CI: 0.06 to 0.35 ), and knowledge ( $\beta$ : $0.09 ; 95 \%$ CI: 0.01 to 0.18 ) and being an ethnic minority was negatively associated with wearing a face mask ( $\beta$ : -0.32 ; $95 \% \mathrm{CI}:-0.60$ to -0.03$)$. Determinants of maintaining social distancing included cue to action ( $\beta$ : $0.19 ; 95 \%$ CI: 0.01 to 0.38$)$ and self-efficacy $(\beta: 0.43$; $95 \%$ CI: 0.19 to 0.66$)$.

\section{DISCUSSION}

This study found that respondents had a medium level of knowledge of COVID-19 and the majority of them had heard of the disease through websites and social media. In comparison, a previous study evaluating parents' knowledge of the $\mathrm{H} 1 \mathrm{~N} 1$ vaccine found that $66 \%$ perceived their knowledge of the H1N1 vaccine to be insufficient at best (16). The difference in the level of knowledge might be due to the increasing amount of information being disseminated online and therefore, its accessibility to young adults in this digital era as evidenced by the main sources of information. Despite having prompt awareness of the disease through the internet, we found that not all of respondents' knowledge of COVID-19 was correct. At the time of this study, the majority of our respondents accurately identified droplets and close contact as the main modes of viral transmission, that we have a test kit to test for the disease, that approved medications to treat the disease are not available, and that a vaccine to prevent the disease is not available. However, some respondents held incorrect beliefs about the disease. The WHO identifies that the virus is mainly transmitted by close contact and respiratory droplets produced through the cough or sneeze of an infected person (6). During the study period laboratory tests exist to screen for the disease, however, no approved pharmacological therapy was available (19). Off-label use of some investigational therapies, such as lopinavir with ritonavir-boost, remdesivir, and herb medicine, have been reported with variable efficacy in the literature (20-24). Additionally, few respondents have also incorrectly identified that a vaccine is available for the disease, although robust research effort in vaccine development is underway (25). Our respondents' knowledge of COVID-19 indicated that although the internet can be an effective way of promoting information, the accuracy of medical information is variable. Only $39 \%$ of online medical information is found to be accurate (26). Our findings highlight the importance of increasing education to young adults on the correct modes of transmission and the rapid dissemination of reliable information on the availability of screening and treatment strategies for this disease.

The HBM was used in this study to identify determinants of preventive health behaviors, including washing hands, wearing a face mask, and maintaining social distancing, recommended by China CDC. For wearing a face mask, this study identified cue to action, self-efficacy, and knowledge as significant predictors. Our finding that cue to action was a significant predictor of face mask-wearing was similar to previous studies during the SARS and H1N1 outbreaks $(12,13,15)$. Cue to action which significantly predicted face mask-wearing in previous studies included recommendations from local government and family members, being in crowded places and places of vulnerability (e.g., hospitals), situational cues (e.g., seeing people coughing or sneezing, seeing others wearing face masks), and availability of face masks (e.g., availability of face masks provided at no cost) $(12,13,15)$. Self-efficacy and knowledge were not always assessed in previous studies. However, when included, they were not significantly correlated with face mask-wearing during the SARS and H1N1 outbreaks $(13,15)$. This study did not find perceived susceptibility as a significant predictor of face mask-wearing, whereas this was consistently identified as a predictor during the SARS and H1N1 outbreaks $(12,13,15)$. This discrepancy could be explained by our respondents' perception that they were at low risk of contracting COVID-19. Since previous studies linking perceived susceptibility to face mask-wearing have found that individuals who feel that they were more vulnerable to the disease were more likely to wear a face mask compared to those who perceived a lower susceptibility to the disease $(12,15)$. In terms of hand washing, we found cue to action, self-efficacy, and knowledge to be significant predictors, and in terms of social distancing, we found cue to action and self-efficacy to be significant predictors. Previous studies have only assessed preventive health behaviors as a whole and have not studied 
TABLE 3 | Multivariate linear regressions on preventive health behaviors.

\begin{tabular}{|c|c|c|c|c|c|c|c|c|c|}
\hline & \multicolumn{3}{|c|}{ Washing hands } & \multicolumn{3}{|c|}{ Wearing a face mask } & \multicolumn{3}{|c|}{ Maintaining social distancing } \\
\hline & $\beta$ & & & $\beta$ & & & $\beta$ & & \\
\hline \multicolumn{10}{|l|}{ Health Belief Model } \\
\hline Perceived susceptibility & 0.03 & -0.06 & 0.11 & 0.00 & -0.05 & 0.04 & 0.08 & -0.05 & 0.21 \\
\hline Perceived severity & 0.01 & -0.08 & 0.11 & 0.00 & -0.05 & 0.05 & 0.04 & -0.10 & 0.18 \\
\hline Perceived benefits & 0.02 & -0.10 & 0.13 & 0.04 & -0.02 & 0.10 & 0.10 & -0.15 & 0.34 \\
\hline Perceived barriers & -0.01 & -0.10 & 0.07 & -0.01 & -0.06 & 0.03 & -0.02 & -0.15 & 0.12 \\
\hline Cue to action & 0.27 & 0.05 & 0.48 & 0.20 & 0.06 & 0.34 & 0.19 & 0.01 & 0.38 \\
\hline Self-efficacy & 0.56 & 0.39 & 0.73 & 0.21 & 0.06 & 0.35 & 0.43 & 0.19 & 0.66 \\
\hline Knowledge & 0.15 & 0.01 & 0.31 & 0.09 & 0.01 & 0.18 & 0.01 & -0.22 & 0.23 \\
\hline \multicolumn{10}{|l|}{ Demographics } \\
\hline \multicolumn{10}{|l|}{ Age } \\
\hline $18-22$ & Ref & & & Ref & & & Ref & & \\
\hline $23+$ & 0.09 & -0.20 & 0.38 & 0.00 & -0.16 & 0.16 & -0.33 & -0.76 & 0.11 \\
\hline \multicolumn{10}{|l|}{ Gender } \\
\hline Female & Ref & & & Ref & & & Ref & & \\
\hline Male & -0.35 & -0.68 & -0.03 & -0.04 & -0.21 & 0.14 & -0.12 & -0.61 & 0.38 \\
\hline \multicolumn{10}{|l|}{ Ethnicity } \\
\hline Han & Ref & & & Ref & & & Ref & & \\
\hline Minority & -0.18 & -0.71 & 0.34 & -0.32 & -0.60 & -0.03 & -0.60 & -1.37 & 0.18 \\
\hline \multicolumn{10}{|l|}{ Residence area } \\
\hline Low-endemic area & Ref & & & Ref & & & Ref & & \\
\hline High-endemic area & -0.14 & -0.46 & 0.18 & -0.07 & -0.24 & 0.10 & 0.09 & -0.38 & 0.56 \\
\hline
\end{tabular}

predictors of individual health behaviors of hand washing and social distancing separately. By identifying predictors of these specific preventive health behaviors, our results could facilitate more targeted educational efforts to enhance the potential adoption of these health behaviors.

Respondents reported that they were moderately or extremely likely to adopt preventive health behaviors. The likelihood to adopt these health behaviors was strongest with face maskwearing (rated extremely likely), followed by social distancing and hand washing (both rated moderately likely). This might be because respondents perceive face mask-wearing as a social norm and a form of civic responsibility, as illustrated in a previous study conducted in Hong Kong during the SARS outbreak (27). Not wearing face masks during the SARS outbreak reportedly led to stigmatization, social seclusion, and discrimination (27). The adverse social consequences of not wearing a face mask, particularly with its visibility, likely serve as a stronger motivator for its use compared to less visible health behaviors such as hand washing. Based on our identified predictors of these behaviors, improving social distancing and hand washing could be achieved by having local governments and/or public health officials exercise cues to action by recommending young adults adopt these measures through websites or social media. These messages should also aim to increase their confidence in adopting these measures in order to improve self-efficacy. Knowledge also significantly predicted washing hands, but not to practice social distancing.
Therefore, to further improve hand hygiene among young adults, public health officials can consider creating online videos and/or infographics illustrating the importance of and the methods to proper hand washing.

Pharmacy students were still staying at home and learning online as all colleges and universities were closed during the study period. This study found that respondents were moderately or extremely likely to adopt preventive health behaviors. The high likelihood of engaging individual preventive measures could support the reopening of colleges and universities in China. Special attention should be paid to washing hands as this is not as visible compared to wearing a face mask. In addition, maintaining social distancing might be challenging in the classroom and cafeteria when college students are back to school. Given the medium level of knowledge of COVID-19 in pharmacy students, continued education through the internet is important. Local and school authorities should make clear guidance to support individuals to adopt preventive health behaviors to reduce the risk of community transmission of COVID-19 in colleges and universities.

This study has some limitations worth mentioning. First, this study only included pharmacy students, therefore the results cannot be generalizable to the general public. Second, due to the use of a questionnaire for data collection, the actual level of knowledge and preventive health behaviors might be different from what has been reported. Third, because only components 
of the HBM were evaluated, there might be other factors that could motivate health behaviors that were not accounted for in this study. However, the HBM is one of the most widely used belief-based psychosocial theories and has been extensively used to assess preventive health behaviors in previous virus outbreaks.

\section{CONCLUSIONS}

This study found that future pharmacists in high and low-endemic areas in China had a medium level of knowledge of COVID-19. Further efforts to improve their knowledge can be focused on providing timely, reliable, and accurate information on the internet. In the HBM, cue to action, self-efficacy, and knowledge were significant predictors of preventive health behaviors of washing hands and wearing a face mask, whereas only cue to action and self-efficacy were significant predictors of maintaining social distancing. To potentially improve the adoption of face mask-wearing, hand washing, and social distancing as appropriate individual preventive measures, targeted educational efforts should be implemented to enhance cue to action, self-efficacy, and knowledge. To prepare for reopening schools, continued monitoring of individuals' knowledge and behavioral responses to COVID-19 is warranted.

\section{REFERENCES}

1. World Health Organization. Rolling Updates on Coronavirus Disease (COVID19). Available online at: https://www.who.int/emergencies/diseases/novelcoronavirus-2019/events-as-they-happen

2. Ji T, Chen HL, Xu J, Wu LN, Li JJ, Chen K, et al. Lockdown contained the spread of 2019 novel coronavirus disease in Huangshi city, China: early epidemiological findings. Clin Infect Dis. (2020) 71:1454-60. doi: $10.1093 / \mathrm{cid} / \mathrm{ciaa} 390$

3. Lau H, Khosrawipour V, Kocbach P, Mikolajczyk A, Schubert J, Bania J, et al. The positive impact of lockdown in Wuhan on containing the COVID-19 outbreak in China. J Travel Med. (2020) 27:taaa037. doi: 10.1093/jtm/taaa037

4. Bayham J, Fenichel EP. Impact of school closures for COVID-19 on the US health-care workforce and net mortality: a modelling study. Lancet Publ. Health. (2020) 5:E271-8. doi: 10.1101/2020.03.09.20033415

5. Chinazzi M, Davis JT, Ajelli M, Gioannini C, Litvinova M, Merler S, et al. The effect of travel restrictions on the spread of the 2019 novel coronavirus (COVID-19) outbreak. Science. (2020) 368:395-400. doi: 10.1101/2020.02.09.20021261

6. World Health Organization. Coronavirus Disease (COVID-19) Advice for the Public. Available online at: https://www.who.int/emergencies/diseases/novelcoronavirus-2019/advice-for-public

7. Centers for Disease Control and Prevention. How to Protect Yourself and Others. Available online at: https://www.cdc.gov/coronavirus/2019-ncov/ prevent-getting-sick/prevention.html

8. Chinese Center for Disease Control and Prevention. Questions on Protection (En). Available online at: http://www.chinacdc.cn/en/COVID19/202002/ P020200310326619051741.pdf

9. Rosenstock IM, Strecher VJ, Becker MH. Social learning theory and the Health Belief Model. Health Educ Q. (1988) 15:175-83. doi: $10.1177 / 109019818801500203$

10. Buglar ME, White KM, Robinson NG. The role of self-efficacy in dental patients' brushing and flossing: testing an extended Health Belief Model. Patient Educ Couns. (2010) 78:269-72. doi: 10.1016/j.pec.2009.06.014

11. Bayat F, Shojaeezadeh D, Baikpour M, Heshmat R, Baikpour M, Hosseini M. The effects of education based on extended health belief model in type

\section{DATA AVAILABILITY STATEMENT}

The raw data supporting the conclusions of this article will be made available by the authors, without undue reservation.

\section{ETHICS STATEMENT}

The studies involving human participants were reviewed and approved by University of Tennessee Health Science Center. The ethics committee waived the requirement of written informed consent for participation.

\section{AUTHOR CONTRIBUTIONS}

GL and JY: conceptualization, methodology, and writing, SH: methodology and writing, RS: methodology and analysis, ML: conceptualization, methodology, analysis, writing, and supervision. All authors contributed to the article and approved the submitted version.

\section{ACKNOWLEDGMENTS}

The authors thank all participants of this study.

2 diabetic patients: a randomized controlled trial. J Diabetes Metab Disord. (2013) 12:45. doi: 10.1186/2251-6581-12-45

12. Tang CS, Wong CY. Factors influencing the wearing of facemasks to prevent the severe acute respiratory syndrome among adult Chinese in Hong Kong. Prevent Med. (2004) 39:1187-93. doi: 10.1016/j.ypmed.2004.04.032

13. Wong CY, Tang CS. Practice of habitual and volitional health behaviors to prevent severe acute respiratory syndrome among Chinese adolescents in Hong Kong. J Adolesc Health. (2005) 36:193-200. doi: 10.1016/j.jadohealth.2004.02.024

14. Jeong HS, Lee DW, Youn CH, Lee MK, Lee SJ, Suh YS, et al. Perception and performance of preventive behaviors for the pandemic influenza in hospital employees and outpatients. Yonsei Med J. (2011) 52:181-7. doi: 10.3349/ymj.2011.52.1.181

15. Zhang CQ, Chung PK, Liu JD, Chan DKC, Hagger MS, Hamilton K. Health beliefs of wearing facemasks for influenza A/H1N1 prevention: a qualitative investigation of Hong Kong older adults. Asia Pac J Public Health. (2019) 31:246-56. doi: 10.1177/1010539519844082

16. Wu CS, Kwong EW, Wong HT, Lo SH, Wong AS. Beliefs and knowledge about vaccination against AH1N1pdm09 infection and uptake factors among Chinese parents. Int J Environ Res Public Health. (2014) 11:1989-2002. doi: 10.3390/ijerph110201989

17. Nowrouzi-Kia B, McGeer A. External cues to action and influenza vaccination among post-graduate trainee physicians in Toronto, Canada. Vaccine. (2014) 32:3830-4. doi: 10.1016/j.vaccine.2014.04.067

18. Wong LP, Alias H, Hassan J, AbuBakar S. Attitudes towards Zika screening and vaccination acceptability among pregnant women in Malaysia. Vaccine. (2017) 35:5912-7. doi: 10.1016/j.vaccine.2017.08.074

19. Li Z, Yi Y, Luo X, Xiong N, Liu Y, Li S, et al. Development and clinical application of a rapid IgM-IgG combined antibody test for SARS-CoV-2 infection diagnosis. J Med Virol. (2020) 92:1518-24. doi: 10.1002/jmv.25727

20. Cao B, Wang Y, Wen D, Liu W, Wang J, Fan G, et al. A trial of lopinavirritonavir in adults hospitalized with severe covid-19. N Engl J Med. (2020) 382:1787-99. doi: 10.1056/NEJMoa2001282

21. Deng L, Li C, Zeng Q, Liu X, Li X, Zhang H, et al. Arbidol combined with $\mathrm{LPV} / \mathrm{r}$ versus LPV/r alone against Corona Virus Disease 2019: a retrospective cohort study. J Infect. (2020) 81:e1-5. doi: 10.1016/j.jinf.2020.03.002 
22. Lu H. Drug treatment options for the 2019-new coronavirus (2019-nCoV). Biosci Trends. (2020) 14:69-71. doi: 10.5582/bst.2020.01020

23. Holshue ML, DeBolt C, Lindquist S, Lofy KH, Wiesman J, Bruce H, et al. First case of 2019 novel coronavirus in the United States. N Engl J Med. (2020) 382:929-36. doi: 10.1056/NEJMoa2001191

24. Ren JL, Zhang AH, Wang XJ. Traditional Chinese medicine for COVID-19 treatment. Pharmacol Res. (2020) 155:104743. doi: 10.1016/j.phrs.2020.104743

25. Fauci AS, Lane HC, Redfield RR. Covid-19 - navigating the uncharted. N Engl J Med. (2020) 382:1268-9. doi: 10.1056/NEJMe2002387

26. Scullard P, Peacock C, Davies P. Googling children's health: reliability of medical advice on the internet. Arch Dis Child. (2010) 95:580-2. doi: $10.1136 /$ adc.2009.168856

27. Siu JY. Qualitative study on the shifting sociocultural meanings of the facemask in Hong Kong since the severe acute respiratory syndrome (SARS) outbreak: implications for infection control in the post-SARS era. Int J Equity Health. (2016) 15:73. doi: 10.1186/s12939-016-0358-0

Conflict of Interest: The authors declare that the research was conducted in the absence of any commercial or financial relationships that could be construed as a potential conflict of interest.

Copyright (c) 2021 Lv, Yuan, Hsieh, Shao and Li. This is an open-access article distributed under the terms of the Creative Commons Attribution License (CC BY).

The use, distribution or reproduction in other forums is permitted, provided the original author(s) and the copyright owner(s) are credited and that the original publication in this journal is cited, in accordance with accepted academic practice. No use, distribution or reproduction is permitted which does not comply with these terms. 